\title{
Generalized Swift-Hohenberg and phase-field-crystal equations based on a second-gradient phase-field theory
}

\author{
Luis Espath $(\mathbb{D} \cdot$ Victor M. Calo $\cdot$ Eliot Fried $(\mathbb{D}$
}

Received: 25 May 2020/ Accepted: 13 August 2020/Published online: 7 October 2020

(C) The Author(s) 2020

\begin{abstract}
The principle of virtual power is used derive a microforce balance for a second-gradient phase-field theory. In conjunction with constitutive relations consistent with a free-energy imbalance, this balance yields a broad generalization of the SwiftHohenberg equation. When the phase field is identified with the volume fraction of a conserved constituent, a suitably augmented version of the free-energy imbalance yields constitutive relations which, in conjunction with the microforce balance and the constituent content balance, delivers a broad generalization of the phase-field-crystal equation. Thermodynamically consistent boundary conditions for situations in which the interface between the system and its environment is structureless and cannot support constituent transport are also developed, as are energy decay relations that
\end{abstract}

\footnotetext{
L. Espath

Department of Mathematics, RWTH Aachen University, 52056 Aachen, Germany

e-mail: espath@uq.rwth-aachen.de

V. M. Calo

Department of Applied Geology, Curtin University, Perth, WA, Australia

e-mail: victor.calo@curtin.edu.au

E. Fried $(\bowtie)$

Mathematics, Mechanics, and Materials Unit, Okinawa

Institute of Science and Technology, Onna,

Okinawa 904-0495, Japan

e-mail: eliot.fried@oist.jp
}

ensue naturally from the thermodynamic structure of the theory.

Keywords Continuum theory · Order parameter . Microstructural fields

\section{Mathematics Subject Classification 74A30 . $82 \mathrm{C} 26 \cdot 35 \mathrm{~B} 36$}

\section{Introduction}

The Swift-Hohenberg and phase-field-crystal equations describe a multitude of processes involving spatiotemporal pattern formation. Swift and Hohenberg [1] derived the first of these equations through a weakly nonlinear approximation of the infinite Prandtl number version of the Boussinesq equations about the Rayleigh number corresponding to the onset of convective instabilitiies. Their equation can be formulated as a relaxation, or "gradient flow", law for an energy functional that depends on a scalar order parameter along with its gradient and Laplacian. The resulting evolution equation is parabolic and involves a fourth-order spatial operator. As Burke and Knobloch [2] explain, a distinguishing feature of the Swift-Hohenberg equation that distinguishes it from other pattern forming equations, like the KuramotoSivashinsky equation, but which stem from small wave-number expansions is that it exhibits a finite 
wavenumber instability at onset and, thus, admits stable spatially periodic equilibria. For this reason, it has been used to study disclinations (Golovin and Nepomnyashchy [3]), defects (Galla and Moro [4]), grain boundaries (Boyer and Viñals [5]), oscillons and other patterns in vibrated granular media (Crawford and Riecke [6]), and radiation-induced microstructural features in immiscible alloys (Simone et al. [7]).

Starting from the classical density functional theory for crystallization, as described by Ramakrishnan and Yussouff [8], Elder and Grant [9] derived the phasefield-crystal theory by approximation for the twoparticle correlation function of the melt that enters the free-energy density of the crystal relative (measured relative to the homogeneous melt). The resulting energy functional is identical to that underpinning the Swift-Hohenberg equation. The salient order parameter is, however, a conserved quantity - the atomic number density. The phase-field-crystal equation is thus the conserved counterpart of the Swift-Hohenberg equation. This relationship is completely analogous to that between the Cahn-Hilliard equation and the Allen-Cahn equation. The spatial operator entering this parabolic equation is consequently of sixthorder. Apart from modeling crystal growth and dissolution, the phase-field-crystal equation has been used to study a broad spectrum of phenomena, including plasticity (Stefanovic et al. [10]), pattern formation in liquid crystals (Löwen [11]), epitaxial growth (Gránásy, et al. [12], Yua et al. [13]), glass formation (Berry and Grant [14]), two-dimensional materials like graphene (Seymour and Provatas [15], grain boundary motion, grain rotation, and dislocation reactions in body-centered-cubic bicrystals (Yamanaka et al. [16]), and plastic flow due to dislocation motion (Skaugen et al. [17]). Additionally, as Provatas et al. [18] explain, the phase-field-crystal equation also provides a natural basis for developing multiscale simulation methods.

Our goal in this paper is to develop a broadly applicable thermodynamically consistent framework that subsumes the Swift-Hohenberg and phase-fieldcrystal equations, accompanied by suitable boundary conditions and associated relations that guarantee that appropriate measures of total energy be nonincreasing along solution paths. To achieve this, we exploit and extend an approach used by Fried and Gurtin [19] to derive generalizations of the scalar Ginzburg-Landau equation and later applied by Gurtin [20] to derive generalized versions of the Cahn-Hilliard equation. In that approach, the phase field is a basic kinematical descriptor and power expenditures associated with its temporal variations, along with those of its spatial gradient, are ascribed to a system of microforces subject to their own balance. However, because the spatial operators entering the Swift-Hohenberg and phase-field-crystal equations are respectively two orders higher than those entering Ginzberg-Landau and Cahn-Hilliard equations, we must also account for power expenditures associated with temporal variations of the second spatial gradient of the phase field. This leads to a second-gradient phase-field theory.

Instead of the pivotal microforce balance of Fried and Gurtin [19] and Gurtin [20], we emulate Gurtin [21] and base our theory on a power balance. This fundamental postulate requires that the instantaneous power expended by all agencies external to any part of the region within which the phase field is defined be equal to the instantaneous power expended by all internal actions within that part. In line with the presence of the second gradient of the phase field, our theory includes a hypermicrostress and a hypermicrotraction above and beyond the microstress, its associated microtraction, and the internal and external microforce densities that enter the conventional firstgradient phase-field theory. We also relax the standard requirement that the microtraction distributed on a surface separating two parts depends only on the orientation of that surface. As consequences of the principle of virtual-power, we obtain a generalization of the pointwise microforce balance from the conventional first-gradient phase-field theory together with representations for the microtraction and the hypermicrotraction. The microstress and hypermicrostress both appear in the generalized microforce balance. Whereas the microtraction depends on surface orientation and surface curvature, the hypermicrotraction depends only on surface orientiation, albeit quadratically. As corollaries of the generalized pointwise microforce balance and the representations for the microtraction and hypermicrotraction, we derive partwise statements of microforce balance and hypermicroforce balance.

In our treatment of energetics, we follow the lead of Fried and Gurtin [19] and Gurtin [20] and confine attention to isothermal processes, with the consequence that the first and second laws of thermodynamics combine to yield a free-energy imbalance. 
Using the pointwise version of that imbalance, we find the largest class of smooth, thermodynamically admissible constitutive relations in which the freeenergy density, internal microforce density, microstress, and hypermicrostress serve as dependent constitutive variables and the phase field along with its first and second spatial gradients and its temporal rate serve as independent constitutive variables. Augmenting our microforce balance with those constitutive relations delivers a broad generalization of the SwiftHohenberg equation. For equations of phase-fieldcrystal type, the phase field is identified as the volume fraction of a conserved constituent governed by a transport equation expressing the balance of constituent content. Using a suitably modified version of the free-energy inequality, we find the largest class of smooth, thermodynamically admissible constitutive relations in which the list of dependent variables considered previously is augmented to include the constituent flux while the corresponding list of independent constitutive variables is augmented to include the chemical potential and its spatial gradient but no longer includes the temporal rate of the phase field. The constitutively augmented microforce balance then determines the chemical potential in terms of the phase field and its first, second, third, and fourth spatial gradients. Augmenting the constituent content balance with the constitutive relation for the constituent flux and the expression for the chemical potential then yields a broad generalization of the phase-field-crystal equation.

To derive thermodynamically consistent boundary conditions for our equations, we adapt an approach introduced by Fried and Gurtin [27] and subsequently extended by Duda et al. [28]. We restrict attention to situations where the boundary is unstructured and cannot support constituent transport. As a starting point, we formulate versions of microforce and hypermicroforce balances and the free-energy imbalance for an arbitrary boundary pillbox. For simplicity, we rule out coupling effects and dissipative interactions with the environment. This leads to what Fried and Gurtin [27] call 'zero-dissipation conditions'. In the absence of constituent transport, we find that it is generally permissible to assign the microtraction and hypermicrotraction on the same portion of the boundary. When species transport is present, we find that it is additionally permissible to assign either the constituent flow or the chemical potential across a portion of the boundary. The thermodynamic structure of our theory ensures the existence of energy decay relations for our generalized Swift-Hohenberg and phase-fieldcrystal equations for this class of boundary conditions.

The remainder of this paper is organized as follows: In Sect. 2, we develop expressions for internal and external expenditures that account explicitly for second gradient effects and state the associated power balance. In Sect. 3, we introduce and obtain the consequences of the principle of virtual-power. In Sect. 4, we describe interactions between the system with state characterized by the phase field and its surroundings. In Sect. 5, we pose the free-energy imbalance for situations where the phase field is not a conserved order parameter and use that imbalance to determine thermodynamically admissible constitutive relations for the microstress, hypermicrostress, and internal microforce density in terms of the phase field, its first and second spatial gradients, and its temporal rate. In Sect. 6, we augment the pointwise microforce balance with the constitutive relations obtained in Sect. 5 to arrive at our generalization of the Swift-Hohenberg equation. In Sects. 7 and 8, we consider the consequences of identifying the phase field with the volume fraction of a single, independent, mobile constituent, augment our theory to account for the transport effects, use the free-energy imbalance to determine thermodynamically admissible constitutive relations for the microstress, hypermicrostress, internal microforce density, and constituent flux, and arrive our generalization of the phase-field-crystal equation. In Sect. 9, we develop thermodynamically consistent boundary conditions that are compatible with our framework. In Sect. 10, we establish energy-decay relations for our generalized versions of the SwiftHohenberg and phase-field-crystal equations. Finally, in Sect. 11, we summarize and discuss our primary findings.

\section{Power expenditures}

We consider a medium described by a phase field $\varphi$ defined on a fixed open region $\mathcal{B}$ of three-dimensional point space and some open time-interval. Our aim in this section is to generalize the first-gradient phasefield theory developed by Fried and Gurtin [19] to account explicitly for effects associated with the second gradient $\operatorname{grad}^{2} \varphi$ of $\varphi$. 


\subsection{Internal power}

In the first-gradient phase-field theory, the internal power of a part $\mathcal{P}$ of $\mathcal{B}$ has the form

$$
\int_{\mathcal{P}}(-\pi \dot{\varphi}+\xi \cdot \operatorname{grad} \dot{\varphi}) \mathrm{d} v,
$$

where $\pi$ and $\xi$ are the internal microforce density and the microstress. To incorporate second-gradient effects, we introduce a second-order tensor, the hypermicrostress $\Sigma$, with associated hypermicrostress power $\Sigma \cdot \operatorname{grad}^{2} \dot{\varphi}$, and replace (1) by

$\mathcal{W}_{\text {int }}(\mathcal{P})=\int_{\mathcal{P}}\left(-\pi \dot{\varphi}+\xi \cdot \operatorname{grad} \dot{\varphi}+\Sigma \cdot \operatorname{grad}^{2} \dot{\varphi}\right) \mathrm{d} v$.

Since $\operatorname{grad}^{2} \dot{\varphi}$ is symmetric, $\Sigma$ splits into a sum of active and reactive components,

$$
\left.\begin{array}{l}
\Sigma=\Sigma_{a}+\Sigma_{r}, \\
\Sigma_{a}=\frac{1}{2}\left(\Sigma+\Sigma^{\top}\right), \\
\Sigma_{r}=\frac{1}{2}\left(\Sigma-\Sigma^{\top}\right),
\end{array}\right\}
$$

with $\Sigma_{r}$ being powerless. Whereas $\Sigma_{a}$ can be determined constitutively, $\Sigma_{r}$ is an unknown field which, like the pressure of an incompressible material, must be determined on a problem-by-problem basis.

\subsection{External power}

In the first gradient phase-field theory, the external counterpart of (1) has the form

$$
\int_{\mathcal{S}} \boldsymbol{\xi} \cdot \boldsymbol{n} \dot{\varphi} \mathrm{d} a+\int_{\mathcal{P}} \gamma \dot{\varphi} \mathrm{d} v,
$$

where $\mathcal{S}=\partial \mathcal{P}$ is the boundary of $\mathcal{P}, \boldsymbol{n}$ is the outward unit normal on $\mathcal{S}$, and $\gamma$ is the external microforce density. Consistent with the presence of the hypermicrostress power $\Sigma \cdot \operatorname{grad}^{2} \dot{\varphi}$ in (1), we replace the surface microtraction $\boldsymbol{\xi} \cdot \boldsymbol{n}$ by a general surface microtraction $\xi_{\mathcal{S}}$ which, among other things, need not depend linearly on $\boldsymbol{n}$. Moreover, recognizing that only the scalar normal component

$$
\frac{\partial \dot{\varphi}}{\partial n}=\operatorname{grad} \dot{\varphi} \cdot \boldsymbol{n}
$$

of $\operatorname{grad} \dot{\varphi}$ is independent of $\dot{\varphi}$ on $\mathcal{S}$, we introduce a surface hypermicrotraction $\sigma_{\mathcal{S}}$ and replace (4) by

$\mathcal{W}_{\text {ext }}(\mathcal{P})=\int_{\mathcal{S}}\left(\xi_{\mathcal{S}} \dot{\varphi}+\sigma_{\mathcal{S}} \frac{\partial \dot{\varphi}}{\partial n}\right) \mathrm{d} a+\int_{\mathcal{P}} \gamma \dot{\varphi} \mathrm{d} v$.

\subsection{Balance of power}

The central hypothesis of our theory is the assertion that the instantaneous internal and external power expenditures are balanced in every process. Precisely, we stipulate that the equality

$$
\mathcal{W}_{\text {ext }}(\mathcal{P})=\mathcal{W}_{\text {int }}(\mathcal{P})
$$

holds at any time and for any choice of $\mathcal{P}$. Although $\Sigma_{r}$ does not enter the expression (3) for $\mathcal{W}_{\text {int }}(\mathcal{P})$, we refrain from assuming that $\Sigma$ is symmetric. Instead, we demonstrate that the final results of our analysis, which include a pointwise statement of microforce balance and representations for the microtraction $\xi_{\mathcal{S}}$ and the hypermicrotraction $\sigma_{\mathcal{S}}$, involve only $\boldsymbol{\Sigma}_{a}$.

\section{Principle of virtual-power}

To formulate the principle of virtual-power, we assume that, at some arbitrarily chosen, but fixed, time, the internal microforce density $\pi$, microstress $\xi$, hypermicrostress $\Sigma$, external microforce density $\gamma$, surface microtraction $\xi_{\mathcal{S}}$, and surface hypermicrotraction $\sigma_{\mathcal{S}}$ are known, but not necessarily independent, fields and consider $\dot{\varphi}$ as a virtual velocity $\chi$ that can be specified independently of any actual process. Then, defining associated reckonings,

$$
\mathcal{V}_{\text {int }}(\mathcal{P} ; \chi)=\int_{\mathcal{P}}\left(-\pi \chi+\xi \cdot \operatorname{grad} \chi+\Sigma \cdot \operatorname{grad}^{2} \chi\right) \mathrm{d} \nu
$$

and

$$
\mathcal{V}_{\text {ext }}(\mathcal{P} ; \chi)=\int_{\mathcal{S}}\left(\xi_{\mathcal{S}} \chi+\sigma_{\mathcal{S}} \frac{\partial \chi}{\partial n}\right) \mathrm{d} a+\int_{\mathcal{P}} \gamma \chi \mathrm{d} v,
$$

of the virtual internal and external power expenditures of a part $\mathcal{P}$ of $\mathcal{B}$, we emulate Gurtin [21] by imposing the requirement that $\pi, \xi, \Sigma, \gamma, \xi_{\mathcal{S}}$, and $\sigma_{\mathcal{S}}$ be consistent with the principle of virtual-power 


$$
\mathcal{V}_{\text {ext }}(\mathcal{P} ; \chi)=\mathcal{V}_{\text {int }}(\mathcal{P} ; \chi)
$$

for all choices of $\mathcal{P}$ and $\chi$. Granted that the collection of all virtual velocities contains all actual, or realizable, velocities, since $\mathcal{V}_{\text {int }}(\mathcal{P} ; \dot{\varphi})=\mathcal{W}_{\text {int }}(\mathcal{P})$ and $\mathcal{V}_{\text {ext }}(\mathcal{P} ; \dot{\varphi})=\mathcal{W}_{\text {ext }}(\mathcal{P})$ the balance of power (7) is a consequence of the principle (10) of virtual-power for $\chi=\dot{\varphi}$. Although it is certainly possible to build a theory on the basis of (10) without having first introduced $\mathcal{W}_{\text {int }}(\mathcal{P})$ and $\mathcal{W}_{\text {ext }}(\mathcal{P})$, the approach taken in Sect. 2 affords the opportunity to describe precisely the power-conjugate pairings that enter the internal and external power expenditures (2) and (6).

The seminal treatments of the principle of virtual power for elastic materials with couple stress and concomitant constitutive dependence on the second gradient of the deformation were advanced by Toupin [22, 23] and Germain [24]. For additional perspective on the principle, see Antman and Osborn [25] and Del Piero [26].

\subsection{Alternative form of the virtual power balance}

To extract useful information from the principle of virtual power, we recast the virtual internal power (8) in a more useful form. Using the identities

$$
\left.\begin{array}{c}
\xi \cdot \operatorname{grad} \chi=\operatorname{div}(\chi \xi)-\chi \operatorname{div} \xi \\
\Sigma \cdot \operatorname{grad}^{2} \chi=\operatorname{div}\left(\Sigma^{\top} \operatorname{grad} \chi-\chi \operatorname{div} \Sigma\right) \\
+\chi \operatorname{div} \operatorname{div} \Sigma,
\end{array}\right\}
$$

and the divergence theorem, we first obtain an intermediate alternative representation for the internal power:

$$
\begin{aligned}
\mathcal{V}_{\text {int }}(\mathcal{P} ; \chi)= & \int_{\mathcal{S}}((\boldsymbol{\xi}-\operatorname{div} \boldsymbol{\Sigma}) \cdot \boldsymbol{n} \chi+\boldsymbol{\Sigma} \boldsymbol{n} \cdot \operatorname{grad} \chi) \mathrm{d} a \\
& -\int_{\mathcal{P}}(\operatorname{div}(\boldsymbol{\xi}-\operatorname{div} \boldsymbol{\Sigma})+\pi) \chi \mathrm{d} v .
\end{aligned}
$$

As a consequence of the decomposition

$$
\operatorname{grad} \chi=\operatorname{grad}_{\mathcal{s}} \chi+\frac{\partial \chi}{\partial n} \boldsymbol{n}
$$

of $\operatorname{grad} \chi$ on $\mathcal{S}$ and the identity

$\boldsymbol{\Sigma} \boldsymbol{n} \cdot \operatorname{grad}_{\mathcal{S}} \chi=\boldsymbol{P} \Sigma \boldsymbol{n} \cdot \operatorname{grad}_{\mathcal{S}} \chi$,

where

$$
\boldsymbol{P}=\boldsymbol{1}-\boldsymbol{n} \otimes \boldsymbol{n}
$$

is the perpendicular projector onto the tangent space of $\mathcal{S}$, we next see that

$$
\begin{aligned}
\int_{\mathcal{S}} \boldsymbol{\Sigma} \boldsymbol{n} \cdot \operatorname{grad} \chi \mathrm{d} a \\
\quad=\int_{\mathcal{S}}\left(\boldsymbol{P} \boldsymbol{\Sigma} \boldsymbol{n} \cdot \operatorname{grad}_{\mathcal{S}} \chi+\boldsymbol{n} \cdot \boldsymbol{\Sigma} \boldsymbol{n} \frac{\partial \chi}{\partial n}\right) \mathrm{d} a .
\end{aligned}
$$

Recalling that, for a tangential vector field $\boldsymbol{f}$ defined on a surface $\mathcal{A}$ with unit outward tangent-normal $v$, the surface divergence theorem takes the form

$$
\int_{\partial \mathcal{A}} \boldsymbol{f} \cdot \boldsymbol{v} \mathrm{d} s=\int_{\mathcal{A}} \operatorname{div}_{\mathcal{A}} f \mathrm{~d} a,
$$

while bearing in mind that the surface $\mathcal{S}$ is closed, whereby $\partial \mathcal{S}$ is empty, and that

$\boldsymbol{P \Sigma \boldsymbol { n }} \cdot \operatorname{grad}_{\mathcal{S}} \chi=\operatorname{div}_{\mathcal{S}}(\chi \boldsymbol{P} \Sigma \boldsymbol{n})-\operatorname{div}_{\mathcal{S}}(\boldsymbol{P} \Sigma \boldsymbol{n}) \chi$,

we find that

$$
\int_{\mathcal{S}} \boldsymbol{P} \Sigma \boldsymbol{n} \cdot \operatorname{grad}_{\mathcal{S}} \chi \mathrm{d} a=-\int_{\mathcal{S}} \operatorname{div}_{\mathcal{S}}(\boldsymbol{P} \Sigma \boldsymbol{n}) \chi \mathrm{d} a
$$

and, thus, that (16) is equivalent to

$$
\begin{aligned}
\int_{\mathcal{S}} \boldsymbol{\Sigma} \boldsymbol{n} \cdot \operatorname{grad} \chi \mathrm{d} a \\
\quad=\int_{\mathcal{S}}\left(\operatorname{div}_{\mathcal{S}}(\boldsymbol{P} \Sigma \boldsymbol{n}) \chi+\boldsymbol{n} \cdot \boldsymbol{\Sigma} \boldsymbol{n} \frac{\partial \chi}{\partial n}\right) \mathrm{d} a .
\end{aligned}
$$

Using (20) in (12), we obtain the desired recasting of the virtual internal power (8):

$$
\begin{aligned}
\mathcal{V}_{\mathrm{int}}(\mathcal{P} ; \chi)= & \int_{\mathcal{S}}\left((\boldsymbol{\xi}-\operatorname{div} \boldsymbol{\Sigma}) \cdot \boldsymbol{n}-\operatorname{div}_{\mathcal{S}}(\boldsymbol{P} \boldsymbol{\Sigma} \boldsymbol{n})\right) \chi \mathrm{d} a \\
& +\int_{\mathcal{S}} \boldsymbol{n} \cdot \boldsymbol{\Sigma} \boldsymbol{n} \frac{\partial \chi}{\partial n} \mathrm{~d} a \\
& -\int_{\mathcal{P}}(\operatorname{div}(\boldsymbol{\xi}-\operatorname{div} \boldsymbol{\Sigma})+\pi) \chi \mathrm{d} v .
\end{aligned}
$$

Finally, augmenting (10) with (9) and (21), we deduce that, given any virtual field $\chi$ and any arbitrary part $\mathcal{P}$, the virtual power balance is satisfied for any arbitrary part $\mathcal{P}$ and any virtual field $\chi$ only if 


$$
\begin{aligned}
& \int_{\mathcal{P}}(\operatorname{div}(\boldsymbol{\xi}-\operatorname{div} \boldsymbol{\Sigma})+\pi+\gamma) \chi \mathrm{d} v \\
& +\int_{\mathcal{S}}\left(\left(\xi_{\mathcal{S}}-(\boldsymbol{\xi}-\operatorname{div} \boldsymbol{\Sigma}) \cdot \boldsymbol{n}+\operatorname{div}_{\mathcal{S}}(\boldsymbol{P} \boldsymbol{\Sigma} \boldsymbol{n})\right) \chi \mathrm{d} a\right. \\
& \quad+\int_{\mathcal{S}}\left(\sigma_{\mathcal{S}}-\boldsymbol{n} \cdot \boldsymbol{\Sigma} \boldsymbol{n}\right) \frac{\partial \chi}{\partial n} \mathrm{~d} a=0 .
\end{aligned}
$$

Reversing the steps leading to the inference (22), we see, conversely, that it implies the virtual-power balance (10).

\subsection{Consequences of the virtual power balance}

\subsubsection{Pointwise microforce balance: representations for the microtraction and the hypermicrotraction}

For any arbitrary part $\mathcal{P}$ and virtual field $\chi$ in (22), we may invoke the fundamental lemma of the calculus of variations to obtain a statement of microforce balance that holds at any point in $\mathcal{B}$ :

$\operatorname{div}(\xi-\operatorname{div} \Sigma)+\pi+\gamma=0$.

Additionally, since the restriction of $\chi$ to $\mathcal{S}$ and its normal derivative $\partial \chi / \partial n$ are kinematically independent and, thus, can be arbitrarily and autonomously chosen at each point of $\mathcal{S}$, we obtain representations for the surface microtraction $\xi_{\mathcal{S}}$ and hypermicrotraction $\sigma_{\mathcal{S}}$ that hold at any smooth point on the boundary $\mathcal{S}$ of a part $\mathcal{P}$ of $\mathcal{B}:$

$$
\left.\begin{array}{l}
\xi_{\mathcal{S}}=(\boldsymbol{\xi}-\operatorname{div} \Sigma) \cdot \boldsymbol{n}-\operatorname{div}_{\mathcal{S}}(\boldsymbol{P} \Sigma \boldsymbol{n}), \\
\sigma_{\mathcal{S}}=\boldsymbol{n} \cdot \boldsymbol{\Sigma} \boldsymbol{n} .
\end{array}\right\}
$$

For $\boldsymbol{\Sigma}=\mathbf{0},(23)$ and $(24)_{1}$ respectively reduce to the pointwise microforce balance $\operatorname{div} \xi+\pi+\gamma=0$ and the representation $\xi_{\mathcal{S}}=\boldsymbol{\xi} \cdot \boldsymbol{n}$ for the microtraction of the first-gradient phase-field theory. In keeping with the corresponding reduction $\sigma_{\mathcal{S}}=0$ of $(24)_{2}$, there is no counterpart of $\sigma_{\mathcal{S}}$ in that theory. Since

$$
\begin{aligned}
\operatorname{div}_{\mathcal{S}}(\boldsymbol{P} \boldsymbol{\Sigma} \boldsymbol{n}) & =\operatorname{div}_{\mathcal{S}}\left(\boldsymbol{\Sigma} \boldsymbol{n}-\sigma_{\mathcal{S}} \boldsymbol{n}\right) \\
& =\boldsymbol{n} \cdot \operatorname{div}_{\mathcal{S}}\left(\boldsymbol{\Sigma}^{\top}\right)-\boldsymbol{\Sigma} \cdot \boldsymbol{L}+2 H \sigma_{\mathcal{S}},
\end{aligned}
$$

where

$$
\boldsymbol{L}=-\operatorname{grad}_{\mathcal{S}} \boldsymbol{n}=-(\operatorname{grad} \boldsymbol{n}) \boldsymbol{P}
$$

is the curvature tensor of $\mathcal{S}$ and $H=\frac{1}{2} \operatorname{tr} \boldsymbol{L}$ is the corresponding mean curvature, we see that $\xi_{\mathcal{S}}$ can be written alternatively as

$$
\xi_{\mathcal{S}}=\left(\boldsymbol{\xi}-\operatorname{div} \boldsymbol{\Sigma}-\operatorname{div}_{\mathcal{S}}\left(\boldsymbol{\Sigma}^{\top}\right)\right) \cdot \boldsymbol{n}+\boldsymbol{\Sigma} \cdot \boldsymbol{L}-2 H \sigma_{\mathcal{S}} .
$$

Thus, noting from $(24)_{2}$ that $\sigma_{\mathcal{S}}$ depends quadratically on the orientation $\boldsymbol{n}$ of $\mathcal{S}$, we deduce that $\xi_{\mathcal{S}}$ is also quadratic in $\boldsymbol{n}$ and depends as well on the curvature tensor $\boldsymbol{L}$ of $\mathcal{S}$. Letting $-\mathcal{S}$ denote the surface $\mathcal{S}$ oriented by $-\boldsymbol{n}$ and noting, with reference to (26), that $-\mathcal{S}$ has curvature tensor $-\boldsymbol{L}$, we thus see that

$\xi_{\mathcal{S}}=-\xi_{\mathcal{S}} \quad$ and $\quad \sigma_{\mathcal{S}}=\sigma_{-\mathcal{S}}$.

The relations (28) represent an action-reaction principle for oppositely oriented surfaces that touch and are tangent at a point.

\subsubsection{Partwise balances for microforces and hypermicroforces}

Integrating $\xi_{\mathcal{S}}$ given by $(24)_{1}$ over the closed surface $\mathcal{S}$ of an arbitrary part $\mathcal{P}$ and using the divergence theorem in conjunction with the pointwise microforce balance (23), we find that

$$
\int_{\mathcal{S}} \xi_{\mathcal{S}} \mathrm{d} a+\int_{\mathcal{S}} \operatorname{div}_{\mathcal{S}}(\boldsymbol{P} \boldsymbol{\Sigma} \boldsymbol{n}) \mathrm{d} a+\int_{\mathcal{P}}(\pi+\gamma) \mathrm{d} \nu=0 .
$$

However, since $\mathcal{S}$ is a closed surface and $\boldsymbol{P} \boldsymbol{\Sigma} \boldsymbol{n}$ is tangential on $\mathcal{S}$, the second integral on the left-hand side of (29) vanishes due to the surface divergence theorem and we, thus, obtain a generalization

$$
\int_{\mathcal{S}} \xi_{\mathcal{S}} \mathrm{d} a+\int_{\mathcal{P}}(\pi+\gamma) \mathrm{d} v=0
$$

the partwise microforce balance from the first-gradient phase-field theory of Fried and Gurtin [19]. If we formally neglect hypermicrostress by setting $\boldsymbol{\Sigma}=\mathbf{0}$, we see that (30) reduces to the partwise microforce balance of the first-gradient phase-field theory. Letting $\boldsymbol{r}=\boldsymbol{x}-\boldsymbol{o}$ denote the vector from an arbitrarily chosen origin $\boldsymbol{o}$ to point in $\boldsymbol{x}$ on $\partial \mathcal{B}$ and integrating the sum $\xi_{\mathcal{S}} \boldsymbol{r}+\sigma_{\mathcal{S}} \boldsymbol{n}$, with $\xi_{\mathcal{S}}$ and $\sigma_{\mathcal{S}}$ given by (24), over $\partial \mathcal{B}$ and again using the divergence theorem in conjunction 
with the pointwise microforce balance (23), we next find that

$$
\begin{aligned}
\int_{\mathcal{S}}\left(\xi_{\mathcal{S}} \boldsymbol{r}+\sigma_{\mathcal{S}} \boldsymbol{n}\right) \mathrm{d} a+\int_{\mathcal{S}} \operatorname{div}_{\mathcal{S}}(\boldsymbol{r} \otimes \boldsymbol{P} \Sigma \boldsymbol{n}) \mathrm{d} a \\
+\int_{\mathcal{P}}(\pi+\gamma) \boldsymbol{r} \mathrm{d} v=\int_{\mathcal{P}} \xi \mathrm{d} v .
\end{aligned}
$$

However, since the second-order tensor $\boldsymbol{r} \otimes \boldsymbol{P} \boldsymbol{\Sigma} \boldsymbol{n}$ is tangential in the sense that it annihilates the normal $\boldsymbol{n}$ to $\mathcal{S}$, we see that the second integral on the left-hand side of (31) vanishes due to the surface divergence theorem and we, thus, obtain a partwise hypermicroforce balance

$$
\int_{\mathcal{S}}\left(\xi_{\mathcal{S}} \boldsymbol{r}+\sigma_{\mathcal{S}} \boldsymbol{n}\right) \mathrm{d} a+\int_{\mathcal{P}}(\pi+\gamma) \boldsymbol{r} \mathrm{d} v=\int_{\mathcal{P}} \xi \mathrm{d} v .
$$

The balance (32) holds trivially in the first-gradient phase-field theory. Indeed, if we set $\Sigma=\mathbf{0}$, so that $\xi_{\mathcal{S}}=\boldsymbol{\xi} \cdot \boldsymbol{n}$ and $\sigma_{\mathcal{S}}=0$, then the first term on the lefthand side of (32) can be rewritten as

$$
\begin{aligned}
\int_{\mathcal{S}}\left(\xi_{\mathcal{S}} \boldsymbol{r}+\sigma_{\mathcal{S}} \boldsymbol{n}\right) \mathrm{d} a & =\int_{\mathcal{S}}(\boldsymbol{r} \otimes \boldsymbol{\xi}) \boldsymbol{n} \mathrm{d} a \\
& =\int_{\mathcal{P}} \operatorname{div}(\boldsymbol{r} \otimes \xi) \mathrm{d} v \\
& =\int_{\mathcal{P}}(\boldsymbol{\xi}+(\operatorname{div} \boldsymbol{\xi}) \boldsymbol{r}) \mathrm{d} v,
\end{aligned}
$$

whereby (32) simplifies to

$$
\int_{\mathcal{P}}(\operatorname{div} \xi+\pi+\gamma) \boldsymbol{r} \mathrm{d} v=0,
$$

which holds trivially due to the pointwise microforce balance $\operatorname{div} \xi+\pi+\gamma=0$ of the first-gradient phasefield theory and therefore contributes no additional information.

\subsection{Symmetry of the hypermicrostress}

Referring to the decomposition (3) of the hypermicrostress $\Sigma$ into a sum $\Sigma=\Sigma_{a}+\Sigma_{r}$ of active and reactive components, the former being symmetric and the latter being skew, we find it convenient to introduce the axial vector field $\varsigma$ associated with $\Sigma_{r}$ and write $\varsigma \times=\Sigma_{r}=\frac{1}{2}\left(\Sigma-\Sigma^{\top}\right)$.

Since $\operatorname{div} \operatorname{div}(\varsigma \times)=-\operatorname{div}(\operatorname{curl} \varsigma)=\mathbf{0}$, we see that $\operatorname{div} \operatorname{div} \boldsymbol{\Sigma}=\operatorname{div} \operatorname{div} \boldsymbol{\Sigma}_{a}$ and, thus, that the pointwise microforce balance (23) is equivalent to

$\operatorname{div}\left(\boldsymbol{\xi}-\operatorname{div} \boldsymbol{\Sigma}_{a}\right)+\pi+\gamma=0$.

Next, since $\operatorname{div}(\varsigma \times)=-\operatorname{curl} \varsigma, \boldsymbol{P}(\varsigma \times) \boldsymbol{n}=\varsigma \times \boldsymbol{n}$, and $\operatorname{div}_{\mathcal{S}}(\boldsymbol{\varsigma} \times \boldsymbol{n})=\boldsymbol{n} \cdot \operatorname{curl} \boldsymbol{\varsigma}$, we see that

$\boldsymbol{n} \cdot \operatorname{div}(\varsigma \times)=-\operatorname{div}_{\mathcal{S}}(\boldsymbol{P}(\varsigma \times) \boldsymbol{n})$

and, thus, that the representation $(24)_{1}$ for the microtraction is equivalent to

$\boldsymbol{\xi}_{\mathcal{S}}=\left(\boldsymbol{\xi}-\operatorname{div} \boldsymbol{\Sigma}_{a}\right) \cdot \boldsymbol{n}-\operatorname{div}_{\mathcal{S}}\left(\boldsymbol{P} \boldsymbol{\Sigma}_{a} \boldsymbol{n}\right)$.

Furthermore, since $\boldsymbol{n} \cdot(\varsigma \times) \boldsymbol{n}=0$, the representation $(24)_{2}$ for the hypermicrotraction is equivalent to

$\sigma_{\mathcal{S}}=\boldsymbol{n} \cdot \boldsymbol{\Sigma}_{a} \boldsymbol{n}$.

Finally, mimicking the steps leading to the partwise microforce and hypermicroforce balances (30) and (32) but using (36) in place of (23) and (38) and (39) in place of (24), we see that those balances hold without change if $\Sigma$ is replaced by $\Sigma_{a}$.

Hence, insofar as the results (23), (24), (30), and (32) of our analysis are concerned, in our initial discussion of the internal power it would have been legitimate to assume that $\Sigma$ be symmetric. Without loss of generality, we therefore assume herafter that:

$$
\Sigma=\Sigma^{\top} \text {. }
$$

\section{Interactions between the system and its surroundings}

Consider an arbitrary subsurface $\mathcal{A}$ of the boundary $\partial \mathcal{B}$ of the region $\mathcal{B}$ occupied by the system and let $\boldsymbol{n}$ denote the outward unit normal on $\partial \mathcal{B}$. Following Fried and Gurtin [27], we view $\mathcal{A}$ as a boundary pillbox, of infinitesimal thickness, that straddles a portion of the boundary. This allows us to isolate the physical processes within on both sides of $\mathcal{A}$. The geometric boundary of the surface $\mathcal{A}$ is simply its edge, the closed curve $\partial \mathcal{A}$. Viewed, in contrast, as a boundary pillbox, $\mathcal{A}$ has parallel faces $\pm \mathcal{A}$ with corresponding unit normals $\pm \boldsymbol{n}$ and a lateral face $\partial \mathcal{A}$, as described by Fried and Gurtin [29]. 
Guided by the partwise microforce and hypermicroforce balances (30) and (32), we introduce environmental tractions $\xi_{\text {env }}$ and $\sigma_{\text {env }}$ and assume that, given any subsurface $\mathcal{A}$ of $\partial \mathcal{B}$,

$\int_{\mathcal{A}} \xi_{\text {env }} \mathrm{d} a$ and $\quad \int_{\mathcal{A}}\left(\xi_{\text {env }} \boldsymbol{r}+\sigma_{\text {env }} \boldsymbol{n}\right) \mathrm{d} a$

represent the net microforce and hypermicroforce exerted by the environment on the portion $\mathcal{A}$ of the pillbox surface. To derive microforce and hypermicroforce balances for the pillbox, we first note that, by (28), the net microforce and net hypermicroforce exerted by the system on the portion $-\mathcal{A}$ of the pillbox surface are

$-\int_{\mathcal{A}} \xi_{\mathcal{S}} \mathrm{d} a \quad$ and $\quad-\int_{\mathcal{A}}\left(\xi_{\mathcal{S}} \boldsymbol{r}+\sigma_{\mathcal{S}} \boldsymbol{n}\right) \mathrm{d} a$.

Referring to (41) $)_{1}$ and $(42)_{1}$, we infer that the microforce balance for $\mathcal{A}$ takes the form

$\int_{\mathcal{A}}\left(\xi_{\text {env }}-\xi_{\mathcal{S}}\right) \mathrm{d} a=0$.

Analogously, referring to $(41)_{2}$ and $(42)_{2}$, we see that the hypermicroforce for $\mathcal{A}$ takes the form

$\int_{\mathcal{A}}\left(\left(\xi_{\text {env }}-\xi_{\mathcal{S}}\right) \boldsymbol{r}+\left(\sigma_{\text {env }}-\sigma_{\mathcal{S}}\right) \boldsymbol{n}\right) \mathrm{d} a=\mathbf{0}$.

Since $\mathcal{A}$ is arbitrary, (43) and (44) localize to yield balances valid pointwise on $\partial \mathcal{B}$ :

$\xi_{\mathrm{env}}=\xi_{\mathcal{S}}, \quad \sigma_{\mathrm{env}}=\sigma_{\mathcal{S}}$.

Finally, invoking the representations (24) for the microstress $\xi$ and hypermicrostress $\Sigma=\Sigma^{\top}$, we find that the pointwise balances $\partial \mathcal{B}$ can be written as

$(\boldsymbol{\xi}-\operatorname{div} \boldsymbol{\Sigma}) \cdot \boldsymbol{n}-\operatorname{div}_{\mathcal{S}}(\boldsymbol{P} \boldsymbol{\Sigma} \boldsymbol{n})=\boldsymbol{\xi}_{\mathrm{env}}$

and

$$
\boldsymbol{n} \cdot \boldsymbol{\Sigma} \boldsymbol{n}=\sigma_{\mathrm{env}} .
$$

\section{Free-energy imbalance: constitutive relations}

We restrict attention to purely mechanical processes governed by the purely mechanical partwise freeenergy imbalance
$\overline{\int_{\mathcal{P}} \psi \mathrm{d} v} \leq \mathcal{W}_{\mathrm{ext}}(\mathcal{P})$

holds for all $\mathcal{P}$, where $\psi$ denotes the free-energy density and $\mathcal{W}_{\text {ext }}(\mathcal{P})$ is given by (6) with $\xi_{\mathcal{S}}$ and $\sigma_{\mathcal{S}}$ is determined according to the representations in (24). Using the power balance (7) in (48), we arrive at the pointwise free-energy imbalance

$$
\dot{\psi}+\pi \dot{\varphi}-\xi \cdot \operatorname{grad} \dot{\varphi}-\Sigma \cdot \operatorname{grad}^{2} \dot{\varphi} \leq 0 .
$$

Guided by the presence of the power conjugate pairings $\pi \dot{\varphi}, \xi \cdot \operatorname{grad} \dot{\varphi}$, and $\Sigma \cdot \operatorname{grad}^{2} \dot{\varphi}$ in (49), we consider a class of constitutive relations that determine $\psi, \pi, \boldsymbol{\xi}$, and $\boldsymbol{\Sigma}=\boldsymbol{\Sigma}^{\top}$ at each point $\boldsymbol{x}$ in $\mathcal{B}$ and each instant $t$ of time as smooth functions of $\varphi, \operatorname{grad} \varphi$, $\operatorname{grad}^{2} \varphi$, and $\dot{\varphi}$ at $(\boldsymbol{x}, t)$. Following Coleman and Noll [30], we allow the external microforce density $\gamma$ to take any value necessary to ensure satisfaction of the microforce balance (23). It can then be shown that the dissipation inequality (49) is satisfied in all processes it is necessary and sufficient to require that:

- The free-energy density $\psi$ is given by a constitutive response function $\hat{\psi}$ that is independent of $\dot{\varphi}$ :

$\psi=\hat{\psi}\left(\varphi, \operatorname{grad} \varphi, \operatorname{grad}^{2} \varphi\right)$.

- The microstress $\xi$ and hypermicrostress $\Sigma$ are given by constitutive response functions $\hat{\xi}$ and $\hat{\Sigma}$ that derive from $\hat{\psi}$ :

$$
\left.\begin{array}{rl}
\boldsymbol{\xi} & =\hat{\boldsymbol{\xi}}\left(\varphi, \operatorname{grad} \varphi, \operatorname{grad}^{2} \varphi\right) \\
& =\frac{\partial \hat{\psi}\left(\varphi, \operatorname{grad} \varphi, \operatorname{grad}^{2} \varphi\right)}{\partial(\operatorname{grad} \varphi)}, \\
\Sigma & =\hat{\boldsymbol{\Sigma}}\left(\varphi, \operatorname{grad} \varphi, \operatorname{grad}^{2} \varphi\right) \\
& =\frac{\partial \hat{\psi}\left(\varphi, \operatorname{grad} \varphi, \operatorname{grad}^{2} \varphi\right)}{\partial\left(\operatorname{grad}^{2} \varphi\right)} .
\end{array}\right\}
$$

- The internal microforce density $\pi$ is given by a constitutive response function $\hat{\pi}$ that splits additively into a contribution derived from $\hat{\psi}$ and a dissipative contribution determined by a nonnegative kinetic modulus $B$ that need not be independent of $\dot{\varphi}$ : 


$$
\begin{aligned}
\pi= & \hat{\pi}\left(\varphi, \operatorname{grad} \varphi, \operatorname{grad}^{2} \varphi, \dot{\varphi}\right) \\
=- & \frac{\partial \hat{\psi}\left(\varphi, \operatorname{grad} \varphi, \operatorname{grad}^{2} \varphi\right)}{\partial \varphi} \\
& -\underbrace{B\left(\varphi, \operatorname{grad} \varphi, \operatorname{grad}^{2} \varphi, \dot{\varphi}\right)}_{\geq 0} \dot{\varphi} .
\end{aligned}
$$

Although the response function $\hat{\psi}$ for the free-energy density $\psi$ serves as a potential for the microstress $\xi$, the hypermicrostress $\Sigma$, and the equilibrium contribution to the internal microforce density $\pi$, the dissipative contribution to $\pi$ is specified through the nonnegative kinetic modulus B. In Sect. 3.3 we showed that if $\Sigma$ includes a skew, reactive component $\Sigma_{r}$, then $\Sigma_{r}$ does not enter the local microforce balance (23) or the representations (24) for the microtraction $\xi_{\mathcal{S}}$ and hypermicrotraction $\sigma_{\mathcal{S}}$. Thus, $\Sigma_{r}$ is completely indeterminate and, consistent with this observation, no generality is incurred by omitting a reactive component from $(51)_{2}$.

\section{Generalized Swift-Hohenberg equation and its specialization}

For each combination of $\hat{\psi}$ and $B$, the local microforce balance (23) and the constitutive relations (51) and (52) for $\xi, \Sigma$, and $\pi$ yield an evolution equation for $\varphi$ :

$$
\begin{gathered}
B\left(\varphi, \operatorname{grad} \varphi, \operatorname{grad}^{2} \varphi, \dot{\varphi}\right) \dot{\varphi} \\
=\operatorname{div}\left\{\frac{\partial \hat{\psi}\left(\varphi, \operatorname{grad} \varphi, \operatorname{grad}^{2} \varphi\right)}{\partial(\operatorname{grad} \varphi)}\right. \\
\left.-\operatorname{div}\left(\frac{\partial \hat{\psi}\left(\varphi, \operatorname{grad} \varphi, \operatorname{grad}^{2} \varphi\right)}{\partial\left(\operatorname{grad}^{2} \varphi\right)}\right)\right\} \\
-\frac{\partial \hat{\psi}\left(\varphi, \operatorname{grad} \varphi, \operatorname{grad}^{2} \varphi\right)}{\partial \varphi}+\gamma .
\end{gathered}
$$

If, in particular, we choose the response function $\hat{\psi}$ and the kinetic modulus $B$ such that

$$
\begin{aligned}
& \hat{\psi}\left(\varphi, \operatorname{grad} \varphi, \operatorname{grad}^{2} \varphi\right) \\
& \quad=f(\varphi)+\frac{1}{2} \lambda\left(\varphi^{2}-2 \ell^{2}|\operatorname{grad} \varphi|^{2}+\ell^{4}|\triangle \varphi|^{2}\right)
\end{aligned}
$$

and

$$
B\left(\varphi, \operatorname{grad} \varphi, \operatorname{grad}^{2} \varphi, \dot{\varphi}\right)=\beta,
$$

where $\Delta$ denotes the Laplacian and $\lambda>0, \ell>0$, and $\beta>0$ are constant material parameters, then the evolution equation (53) specializes to

$\beta \dot{\varphi}=-\lambda\left(1+\ell^{2} \triangle\right)^{2} \varphi-f^{\prime}(\varphi)+\gamma$.

If, moreover, the potential $f$ and the coefficients $\lambda, \ell$, and $\beta$ are chosen suitably, then (56) can be made to coincide with the Swift-Hohenberg [1] equation, in which case $\gamma$ is a source term that accounts for random fluctuations. For this reason, we refer to the progenitor (53) of (56) as the 'generalized Swift-Hohenberg equation.'

\section{Incorporation of constituent transport}

\subsection{Constituent-content balance}

We next extend our formulation to the case where the phase field $\varphi$ represents the mass fraction of a conserved constituent with chemical potential $\mu$, flux $\jmath$, and external supply-rate $s$, per unit volume, while continuing to restrict attention to isothermal processes. Following Gurtin's [20, §3] derivation of the CahnHilliard equation, we therefore supplement the local microforce balance (23) by a partwise constituentcontent balance

$\overline{\int_{\mathcal{P}} \varphi \mathrm{d} v}=-\int_{\mathcal{S}} \jmath \cdot \boldsymbol{n} \mathrm{d} a+\int_{\mathcal{P}} s \mathrm{~d} v$,

the pointwise version of which is

$\dot{\varphi}=-\operatorname{div} \jmath+s$.

To account for constituent transport between the system and its surrounding, we introduce an environmental flux Jenv. The constituent-content balance for the boundary pillbox corresponding to an arbitrary subsurface $\mathcal{A}$ of $\partial \mathcal{B}$ then takes the form

$\int_{\mathcal{A}}\left(\jmath_{\text {env }}+\jmath \cdot \boldsymbol{n}\right) \mathrm{d} a=0$

and localizes to yield the following pointwise condition on $\partial \mathcal{B}$ :

$\jmath_{\mathrm{env}}+\jmath \cdot \boldsymbol{n}=0$. 
7.2 Free-energy imbalance: constitutive relations

To account for the rate at which the total free-energy of $\mathcal{P}$ changes due to constituent transport, we augment the partwise free-energy imbalance (48), giving

$\overline{\int_{\mathcal{P}} \psi \mathrm{d} v} \leq \mathcal{W}_{\text {ext }}(\mathcal{P})-\int_{\mathcal{S}} \mu \jmath \cdot \boldsymbol{n} \mathrm{d} a+\int_{\mathcal{P}} \mu s \mathrm{~d} v$.

Localizing (61) and using the balances (23) and (58) to eliminate the external microforce density $\gamma$ and the rate $s$ of constituent production, we arrive at the pointwise free-energy imbalance

$\dot{\psi}+(\pi-\mu) \dot{\varphi}-\xi \cdot \operatorname{grad} \dot{\varphi}$

$-\Sigma \cdot \operatorname{grad}^{2} \dot{\varphi}+\jmath \cdot \operatorname{grad} \mu \leq 0$.

In recognition of the presence of the power-conjugate pairing $\Sigma \cdot \operatorname{grad}^{2} \dot{\varphi}$ in (62), we augment Gurtin's [20, $\S 3]$ lists $(\varphi, \operatorname{grad} \varphi, \mu, \operatorname{grad} \mu)$ and $(\psi, \xi, \pi, \jmath)$ of independent and dependent constitutive variables to include $\operatorname{grad}^{2} \varphi$ and $\Sigma$, respectively. Restricting attention to smooth constitutive relations, we then find that the local imbalance (62) is satisfied in all processes if and only the free-energy density $\psi$, microstress $\xi$, and microstress $\Sigma$ depend at most on $\left(\varphi, \operatorname{grad} \varphi, \operatorname{grad}^{2} \varphi\right)$ and - as for a nonconserved phase field — are given by (50) and (51), and if:

- The internal microforce $\pi$ is given by a constitutive response function $\hat{\pi}$ that differs from the chemical potential $\mu$ by a contribution derived from the response function $\hat{\psi}$ :

$$
\begin{aligned}
\pi & =\hat{\pi}\left(\varphi, \operatorname{grad} \varphi, \operatorname{grad}^{2} \varphi, \mu\right) \\
& =\mu-\frac{\partial \hat{\psi}\left(\varphi, \operatorname{grad} \varphi, \operatorname{grad}^{2} \varphi\right)}{\partial \varphi} .
\end{aligned}
$$

- The constituent flux $\jmath$ is given by a constitutive response function $\hat{\jmath}$ of the form

$$
\begin{aligned}
\jmath & =\hat{\jmath}\left(\varphi, \operatorname{grad} \varphi, \operatorname{grad}^{2} \varphi, \mu, \operatorname{grad} \mu\right) \\
& =-\boldsymbol{M}\left(\varphi, \operatorname{grad} \varphi, \operatorname{grad}^{2} \varphi, \mu, \operatorname{grad} \mu\right) \operatorname{grad} \mu,
\end{aligned}
$$

where the mobility tensor $\boldsymbol{M}$ must obey the residual dissipation inequality

$\operatorname{grad} \mu \cdot \boldsymbol{M}\left(\varphi, \operatorname{grad} \varphi, \operatorname{grad}^{2} \varphi, \mu, \operatorname{grad} \mu\right) \operatorname{grad} \mu \geq 0$ for all choices of $\varphi, \operatorname{grad} \varphi, \operatorname{grad}^{2} \varphi, \mu$, and $\operatorname{grad} \mu$.

In contrast to the theory previously developed for a nonconserved phase field $\varphi$, the constitutive relation (63) for the internal microforce density $\pi$ is purely conservative. Instead of being tied to kinetics associated with the evolution of $\varphi$, dissipation is in the present context a consequence of constituent transport.

\section{Generalized phase-field-crystal equation and its specialization}

For each choice of $\hat{\psi}$, the local microforce balance (23) and the constitutive relations (51) and (63) for $\xi, \Sigma$, and $\pi$ determines the chemical potential $\mu$ in the form

$$
\begin{aligned}
& \mu= \frac{\partial \hat{\psi}\left(\varphi, \operatorname{grad} \varphi, \operatorname{grad}^{2} \varphi\right)}{\partial \varphi} \\
&-\operatorname{div}\left\{\frac{\partial \hat{\psi}\left(\varphi, \operatorname{grad} \varphi, \operatorname{grad}^{2} \varphi\right)}{\partial(\operatorname{grad} \varphi)}\right. \\
&\left.-\operatorname{div}\left(\frac{\partial \hat{\psi}\left(\varphi, \operatorname{grad} \varphi, \operatorname{grad}^{2} \varphi\right)}{\partial\left(\operatorname{grad}^{2} \varphi\right)}\right)\right\}-\gamma,
\end{aligned}
$$

where the external microforce density $\gamma$ can embody, for instance, the potential energy, per unit volume, of an external gravitational or electromagetic field. The associated evolution equation for $\varphi$ arises from using (66) in the consequence

$$
\dot{\varphi}=\operatorname{div}\left(\boldsymbol{M}\left(\varphi, \operatorname{grad} \varphi, \operatorname{grad}^{2} \varphi, \mu, \operatorname{grad} \mu\right) \operatorname{grad} \mu\right)+s
$$

of augmenting the constituent-content balance (58) with the constitutive relation (64) for the constituent flux $j$. Due to the dependence of the response function $\hat{\psi}$ on $\operatorname{grad}^{2} \varphi, \mu$ as determined by (66) involves fourthorder spatial derivatives of $\varphi$ and (67) thus includes sixth-order spatial derivatives of $\varphi$.

Mimicking the assumptions leading from the generalized Swift-Hohenberg equation (53) to the more familiar specialization (56), we take $\hat{\psi}$ to be of the form (54) and assume that the mobility tensor has the form

$$
\boldsymbol{M}\left(\varphi, \operatorname{grad} \varphi, \operatorname{grad}^{2} \varphi, \mu, \operatorname{grad} \mu\right)=M \mathbf{1},
$$

where $M>0$ is a material parameter. Then, (66) reduces to 


$$
\begin{aligned}
\mu & =f^{\prime}(\varphi)+\lambda\left(\varphi+2 \ell^{2} \Delta \varphi+\ell^{4} \Delta^{2} \varphi\right)-\gamma \\
& =f^{\prime}(\varphi)+\lambda\left(1+\ell^{2} \triangle\right)^{2} \varphi-\gamma
\end{aligned}
$$

and the evolution equation for $\varphi$ that arises on using (68) and (69) takes the form

$$
\dot{\varphi}=M \triangle\left(\lambda\left(1+\ell^{2} \triangle\right)^{2} \varphi+f^{\prime}(\varphi)\right)+\bar{s},
$$

where we have introduced an effective constituent supply rate $\bar{s}=s+M \triangle \gamma$. If, moreover, the potential $f$ and the coefficients $\lambda, \ell$, and $M$ are chosen suitably, then (56) can be made to coincide with the phase-fieldcrystal equation originated by Elder and Grant [9], in which case $\bar{s}$ is a source term that accounts for random fluctuations. For this reason, we refer to the evolution equation that arises from using (66) to eliminate $\mu$ from (67) as the 'generalized phase-field-crystal equation.'

\section{Boundary conditions}

\subsection{Free-energy imbalance for a boundary pillbox}

We next adapt the approach of Fried and Gurtin [27] and Duda et al. [28] to derive thermodynamically consistent conditions on the boundary $\partial \mathcal{B}$ of the region $\mathcal{B}$ that serves as an interface between the system and the environment. For simplicity, we pursue a unified approach in which constituent transport is taken into consideration from the outset and restrict attention to situations where $\partial \mathcal{B}$ is not endowed with surface energy and cannot support constituent transport. Granted these restrictions, the free-energy imbalance for boundary pillbox corresponding to a subsurface $\mathcal{A}$ of $\partial \mathcal{B}$ has the form

$$
\begin{aligned}
\int_{\mathcal{A}}\left(\xi_{\text {env }} \dot{\varphi}_{\text {env }}+\sigma_{\text {env }} \frac{\partial \dot{\varphi}_{\text {env }}}{\partial n}\right) \mathrm{d} a \\
-\int_{\mathcal{A}}\left(\xi_{\mathcal{S}} \dot{\varphi}+\sigma_{\mathcal{S}} \frac{\partial \dot{\varphi}}{\partial n}\right) \mathrm{d} a \\
\quad+\int_{\mathcal{A}} \mu_{\text {env }} \jmath_{\text {env }} \mathrm{d} a+\int_{\mathcal{A}} \jmath \cdot \boldsymbol{n} \mathrm{d} a \geq 0,
\end{aligned}
$$

From the versions $(45)_{1},(45)_{2}$, and (60) of the microforce, hypermicroforce, and constituent-content balances that apply on $\partial \mathcal{B}$ in (71) and taking into account the arbitrary nature of $\mathcal{A}$, we obtain a corresponding inequality,

$$
\begin{aligned}
\left(\dot{\varphi}_{\mathrm{env}}\right. & -\dot{\varphi}) \xi_{\mathrm{env}}+\left(\frac{\partial \dot{\varphi}_{\mathrm{env}}}{\partial n}-\frac{\partial \dot{\varphi}}{\partial n}\right) \sigma_{\mathrm{env}} \\
& +\jmath_{\mathrm{env}}\left(\mu_{\mathrm{env}}-\mu\right) \geq 0,
\end{aligned}
$$

which holds pointwise on $\partial \mathcal{B}$. In general, (46), (47), (60), and (72) can be used to formulate boundary conditions that incorporate coupling between microstructural evolution associated with the phase field and constituent transport, while accounting for dissipative interactions between the system and its surroundings. To ensure that (72) holds, it is sufficient — but certainly not necessary — to require that

$$
\left.\begin{array}{r}
\left(\dot{\varphi}_{\mathrm{env}}-\dot{\varphi}\right) \xi_{\mathrm{env}}=0, \\
\left(\frac{\partial \dot{\varphi}_{\mathrm{env}}}{\partial n}-\frac{\partial \dot{\varphi}}{\partial n}\right) \sigma_{\mathrm{env}}=0, \\
\text { Jenv } \left._{\mathrm{env}}-\mu\right)=0 .
\end{array}\right\}
$$

\subsection{Uncoupled zero-dissipation boundary conditions}

These equalities in (73) are the versions of what Fried and Gurtin [27] call 'uncoupled zero-dissipation conditions' appropriate to the present investigation. The following classes of boundary conditions, posed for an arbitrary subsurface $\mathcal{A}$ of $\partial \mathcal{B}$, are consistent with (46), (47), (60), and (72):

(i) Assigned microtraction on $\mathcal{A}$ : For this class of boundary conditions, $\xi_{\text {env }}$ is given on $\mathcal{A}$ and $\xi_{\mathcal{S}}=(\boldsymbol{\xi}-\operatorname{div} \boldsymbol{\Sigma}) \cdot \boldsymbol{n}-\operatorname{div}_{\mathcal{S}}(\boldsymbol{P} \boldsymbol{\Sigma} \boldsymbol{n}) \quad$ is prescribed on $\mathcal{A}$ in accord with

$$
(\boldsymbol{\xi}-\operatorname{div} \boldsymbol{\Sigma}) \cdot \boldsymbol{n}-\operatorname{div}_{\mathcal{S}}(\boldsymbol{P} \boldsymbol{\Sigma} \boldsymbol{n})=\boldsymbol{\xi}_{\mathrm{env}},
$$

the surfacial microforce balance (46) is trivially satisfied on $\mathcal{A}$, and $\varphi_{\text {env }}$ is determined on $\mathcal{S}$ through (73) $)_{1}$. If, in particular, $\xi_{\text {env }}=0$, then $\mathcal{B}$ is free of microtraction on the subset $\mathcal{A}$ of $\partial \mathcal{B}$.

(ii) Assigned hypermicrotraction on $\mathcal{A}$ : For this class of boundary conditions, $\sigma_{\text {env }}$ is given on $\mathcal{A}$ and $\sigma_{\mathcal{S}}=\boldsymbol{n} \cdot \boldsymbol{\Sigma} \boldsymbol{n}$ is prescribed on $\mathcal{A}$ in accord with

$\boldsymbol{n} \cdot \boldsymbol{\Sigma} \boldsymbol{n}=\sigma_{\mathrm{env}}$, 
the surfacial hypermicroforce balance (47) is trivially satisfied on $\mathcal{A}$, and $\partial \varphi_{\text {env }} / \partial n$ is determined on $\mathcal{A}$ through (73) 2 . If, in particular, $\sigma_{\mathrm{env}}=0$, then $\mathcal{B}$ is free of hypermicrotraction on the subset $\mathcal{A}$ of $\partial \mathcal{B}$.

(iii) Assigned constituent flow across $\mathcal{A}$ : For this class of boundary conditions, $\jmath_{\mathrm{env}}$ is given and $\jmath \cdot \boldsymbol{n}$ is prescribed on $\mathcal{A}$ in accord with

$$
\jmath \cdot \boldsymbol{n}=-\jmath_{\mathrm{env}},
$$

the surfacial constituent-content balance (60) is trivially satisfied on $\mathcal{A}$, and $\mu_{\text {env }}$ is determined on $\mathcal{A}$ through (73) 3 . If, in particular, $J_{\mathrm{env}}=0$, then $\mathcal{B}$ is chemically insulated from the environment on the subset of $\mathcal{A}$ of $\partial \mathcal{B}$.

(iv) Assigned chemical potential on $\mathcal{A}$ : For this class of boundary conditions, $\mu_{\mathrm{env}}$ is given and $\mu$ is prescribed on $\mathcal{S}$ in accord with

$\mu=\mu_{\mathrm{env}}$,

Jenv is determined on $\mathcal{A}$ through the surfacial constituent-content balance (60), and is determined on $\mathcal{A}$ through (73) 3 . If, in particular, $\mu_{\text {env }}=$ constant, then $\mathcal{B}$ is in contact with a reservoir of uniform chemical potential on the subset of $\mathcal{A}$ of $\partial \mathcal{B}$.

Although the microtraction and hypermicrotraction can be assigned on the same portion of $\partial \mathcal{B}$, the constituent flow and the chemical potential can only be assigned on complementary portions of $\partial \mathcal{B}$. The list (i)-(iv) of boundary conditions is not comprehensive. It is also possible to assign $\varphi$ or $\partial \varphi / \partial n$ on $\mathcal{A}$. Physical motivation for such boundary conditions is, however, lacking. We thus exclude them from consideration at this time.

In the absence of constituent transport, (71) reduces to an imbalance involving environmental and internal power expenditures. If the inequality is then replaced by an equality, then the resulting balance of power can be viewed as a counterpart, applicable to a subsurface $\mathcal{A}$ of the boundary $\partial \mathcal{B}$ of $\mathcal{B}$, of the balance of power (7) for a part $\mathcal{P}$ of $\mathcal{B}$. It is of course possible to formulate an associated balance of virtual-power for a subsurface $\mathcal{A}$ of $\partial \mathcal{B}$ and the boundary conditions presented above in items (i) and (ii) can be obtained as a consequence of that virtual balance.
9.3 Constitutively augmented boundary
conditions

We next specialize the (i)-(iv) to develop boundary conditions for the generalized Swift-Hohenberg equation (53) and the generalized phase-field-crystal equation arising from combining (66), (67), and (68).

\subsubsection{Generalized Swift-Hohenberg equation and its specialization}

For the constitutive relations (51) underlying the generalized Swift-Hohenberg equation (53), the boundary conditions (74) and (75) specialize to

$$
\begin{gathered}
\left\{\frac{\partial \hat{\psi}\left(\varphi, \operatorname{grad} \varphi, \operatorname{grad}^{2} \varphi\right)}{\partial(\operatorname{grad} \varphi)}\right. \\
\left.-\operatorname{div}\left(\frac{\partial \hat{\psi}\left(\varphi, \operatorname{grad} \varphi, \operatorname{grad}^{2} \varphi\right)}{\partial\left(\operatorname{grad}^{2} \varphi\right)}\right)\right\} \cdot \boldsymbol{n} \\
\quad-\operatorname{div}_{\mathcal{S}}\left(\boldsymbol{P} \frac{\partial \hat{\psi}\left(\varphi, \operatorname{grad}^{2}, \operatorname{grad}^{2} \varphi\right)}{\partial\left(\operatorname{grad}^{2} \varphi\right)} \boldsymbol{n}\right)=\xi_{\text {env }},
\end{gathered}
$$

and

$\boldsymbol{n} \cdot \frac{\partial \hat{\psi}\left(\varphi, \operatorname{grad} \varphi, \operatorname{grad}^{2} \varphi\right)}{\partial\left(\operatorname{grad}^{2} \varphi\right)} \boldsymbol{n}=\sigma_{\mathrm{env}}$,

respectively. Moreover, for the constitutive relations (51) underlying the classical Swift-Hohenberg equation, (74) and (75) specialize further to

$\lambda \ell^{2}\left(\frac{\partial \varphi}{\partial n}+\ell^{2} \frac{\partial(\Delta \varphi)}{\partial n}\right)=-\xi_{\text {env }}$

and

$\lambda \ell^{4} \Delta \varphi=\sigma_{\text {env }}$.

\subsubsection{Generalized phase-field-crystal equation and its specialization}

In addition to the boundary conditions (78) and (79), for the constitutive relations (63) and (64) underlying the generalized phase-field-crystal Eqs. (66), (67), and (68), the boundary conditions (76) and (77) specialize to 


$$
\begin{aligned}
& M \operatorname{grad}\left\{\frac{\partial \hat{\psi}\left(\varphi, \operatorname{grad} \varphi, \operatorname{grad}^{2} \varphi\right)}{\partial \varphi}\right. \\
& -\operatorname{div}\left\{\frac{\partial \hat{\psi}\left(\varphi, \operatorname{grad} \varphi, \operatorname{grad}^{2} \varphi\right)}{\partial(\operatorname{grad} \varphi)}\right. \\
& \left.\left.-\operatorname{div}\left(\frac{\partial \hat{\psi}\left(\varphi, \operatorname{grad} \varphi, \operatorname{grad}^{2} \varphi\right)}{\partial\left(\operatorname{grad}^{2} \varphi\right)}\right)\right\}-\gamma\right\} \cdot \boldsymbol{n}=-\jmath_{\mathrm{env}}
\end{aligned}
$$

and

$$
\begin{aligned}
& \frac{\partial \hat{\psi}\left(\varphi, \operatorname{grad} \varphi, \operatorname{grad}^{2} \varphi\right)}{\partial \varphi} \\
& -\operatorname{div}\left\{\frac{\partial \hat{\psi}\left(\varphi, \operatorname{grad} \varphi, \operatorname{grad}^{2} \varphi\right)}{\partial(\operatorname{grad} \varphi)}\right. \\
& \left.-\operatorname{div}\left(\frac{\partial \hat{\psi}\left(\varphi, \operatorname{grad} \varphi, \operatorname{grad}^{2} \varphi\right)}{\partial\left(\operatorname{grad}^{2} \varphi\right)}\right)\right\}=\mu_{\mathrm{env}}
\end{aligned}
$$

respectively. Moreover, for the constitutive relations (51), (63) and (64) underlying the classical phasefield-crystal equation, (76) and (77) specialize further to

$$
M \frac{\partial\left(f^{\prime}(\varphi)+\lambda\left(1+\ell^{2} \Delta\right)^{2} \varphi-\gamma\right)}{\partial n}=-\jmath_{\text {env }}
$$

and

$f^{\prime}(\varphi)+\lambda\left(1+\ell^{2} \Delta\right)^{2} \varphi-\gamma=\mu_{\mathrm{env}}$.

\section{Energy-decay relations for uncoupled zero- dissipation boundary conditions}

We next establish energy-decay relations for the generalized Swift-Hohenberg Eq. (53) and the phase-field-crystal equation arising from combining (66) and (67). In so doing, we assume that the assigned microtraction and hypermicrotraction conditions (74) and (75) on all of $\partial \mathcal{B}$ and for the generalized phasefield-crystal equation arising from combining (66) and (67) subject to the assigned microtraction and hypermicrotraction conditions (74) and (75) on all of $\partial \mathcal{B}$. Additionally, we assume either that the assigned constituent flow condition (76) or that the assigned chemical potential condition (77) holds on all of $\partial \mathcal{B}$. In the latter case, we restrict attention to situations in which the system is in contact with a reservoir with uniform chemical potential $\mu_{\text {env }}=$ constant.
Our results rely on identities for the partial timederivative $\psi$ of the free-energy density $\psi$ which we now obtain. To derive those identities, we first observe that, from the relations $(50),(51)_{1}$, and $(51)_{2}$ for the free-energy density $\psi$, microstress $\xi$, and hypermicrostress $\Sigma$ in the constitutive theories in the generalized Swift-Hohenberg equation (53) and the generalized phase-field-crystal equations (66)-(67),

$$
\begin{aligned}
\dot{\psi}= & \frac{\partial \hat{\psi}\left(\varphi, \operatorname{grad} \varphi, \operatorname{grad}^{2} \varphi\right)}{\partial \varphi} \dot{\varphi} \\
& +\frac{\partial \hat{\psi}\left(\varphi, \operatorname{grad} \varphi, \operatorname{grad}^{2} \varphi\right)}{\partial(\operatorname{grad} \varphi)} \cdot \operatorname{grad} \dot{\varphi} \\
& +\frac{\partial \hat{\psi}\left(\varphi, \operatorname{grad} \varphi, \operatorname{grad}^{2} \dot{\varphi}\right)}{\partial\left(\operatorname{grad}^{2} \varphi\right)} \cdot \operatorname{grad}^{2} \dot{\varphi} \\
= & \frac{\partial \hat{\psi}\left(\varphi, \operatorname{grad} \varphi, \operatorname{grad}^{2} \varphi\right)}{\partial \varphi} \dot{\varphi}+\xi \cdot \operatorname{grad} \dot{\varphi}+\Sigma \cdot \operatorname{grad}^{2} \dot{\varphi} \\
= & \left(\frac{\partial \hat{\psi}\left(\varphi, \operatorname{grad} \varphi, \operatorname{grad}^{2} \dot{\varphi}\right)}{\partial \varphi}-\operatorname{div}(\xi-\operatorname{div} \Sigma)\right) \dot{\varphi} \\
& +\operatorname{div}\left(\dot{\varphi}(\xi-\operatorname{div} \Sigma)+\Sigma^{\top} \operatorname{grad} \dot{\varphi}\right) .
\end{aligned}
$$

Using the local microforce balance (23) in (86), we next see that

$$
\begin{aligned}
& \dot{\psi}=(\left.\pi+\frac{\partial \hat{\psi}\left(\varphi, \operatorname{grad} \varphi, \operatorname{grad}^{2} \varphi\right)}{\partial \varphi}+\gamma\right) \dot{\varphi} \\
&+\operatorname{div}\left(\dot{\varphi}(\xi-\operatorname{div} \boldsymbol{\Sigma})+\boldsymbol{\Sigma}^{\top} \operatorname{grad} \dot{\varphi}\right) .
\end{aligned}
$$

The relation (87) holds regardless of whether constituent transport is present. Referring, in particular, to (52), we see that in the absence of constituent transport (87) specializes to

$$
\dot{\psi}=\operatorname{div}\left(\dot{\varphi}(\xi-\operatorname{div} \Sigma)+\Sigma^{\top} \operatorname{grad} \dot{\varphi}\right)+\gamma \dot{\varphi}-B \dot{\varphi}^{2},
$$

where, for brevity, we have suppressed the argument $\left(\varphi, \operatorname{grad} \varphi, \operatorname{grad}^{2} \varphi, \dot{\varphi}\right)$ of the kinetic modulus $B$. Alternatively, referring to (63), we see that in the presence of constituent transport (87) specializes to

$$
\dot{\psi}=\operatorname{div}\left(\dot{\varphi}(\xi-\operatorname{div} \Sigma)+\boldsymbol{\Sigma}^{\top} \operatorname{grad} \dot{\varphi}\right)+\gamma \dot{\varphi}+\mu \dot{\varphi} .
$$

Furthermore, using the pointwise constituent-content balance (58) and the constitutive relation (64) for the constituent flux , we see that (90) is equivalent to 


$$
\begin{aligned}
\dot{\psi}= & \operatorname{div}\left(\dot{\varphi}(\xi-\operatorname{div} \Sigma)+\Sigma^{\top} \operatorname{grad} \dot{\varphi}-\mu \jmath\right) \\
& +\gamma \dot{\varphi}+\mu s-\operatorname{grad} \mu \cdot \boldsymbol{M} \operatorname{grad} \mu,
\end{aligned}
$$

where, for brevity, we have suppressed the argument $\left(\varphi, \operatorname{grad} \varphi, \operatorname{grad}^{2} \varphi, \mu, \operatorname{grad} \mu\right)$ of the mobility tensor M.

\subsection{Generalized Swift-Hohenberg equation}

Integrating (88) over $\mathcal{B}$, applying the transport and divergence theorems and using the boundary conditions (74) and (75) for assigned microtraction and hypermicrotraction in the identity

$$
\begin{aligned}
\dot{\varphi} & (\boldsymbol{\xi}-\operatorname{div} \boldsymbol{\Sigma}) \cdot \boldsymbol{n}+\boldsymbol{\Sigma} \boldsymbol{n} \cdot \operatorname{grad} \dot{\varphi} \\
& =\xi_{\mathrm{env}} \dot{\varphi}+\sigma_{\mathrm{env}} \frac{\partial \dot{\varphi}}{\partial n}+\operatorname{div}_{\mathcal{S}}(\dot{\varphi} \boldsymbol{P} \Sigma \boldsymbol{n}),
\end{aligned}
$$

while taking into considersation that, by the surface divergence theorem and since $\partial \mathcal{B}$ is a closed surface,

$$
\int_{\partial \mathcal{B}} \operatorname{div}_{\mathcal{S}}(\dot{\varphi} \boldsymbol{P} \Sigma \boldsymbol{n}) \mathrm{d} a=0,
$$

we obtain the identity

$$
\begin{aligned}
\overline{\int_{\mathcal{B}} \psi \mathrm{d} v}= & \int_{\partial \mathcal{B}}\left(\xi_{\text {env }} \dot{\varphi}+\sigma_{\text {env }} \frac{\partial \dot{\varphi}}{\partial n}\right) \mathrm{d} a \\
& +\int_{\mathcal{B}} \gamma \dot{\varphi} \mathrm{d} v-\int_{\mathcal{B}} B \dot{\varphi}^{2} \mathrm{~d} v,
\end{aligned}
$$

where the dependence of the kinetic modulus $B$ on $\left(\varphi, \operatorname{grad} \varphi, \operatorname{grad}^{2} \varphi, \dot{\varphi}\right)$ is again suppressed. Recalling that (73) $)_{1,2}$ apply on $\partial \mathcal{B}$ and, from (52), that $B$ must satisfy $B \geq 0$, we thus deduce an energy-decay relation

$$
\overline{\int_{\mathcal{B}} \psi \mathrm{d} v} \leq \int_{\partial \mathcal{B}}\left(\xi_{\text {env }} \dot{\varphi}_{\text {env }}+\sigma_{\text {env }} \frac{\partial \dot{\varphi}_{\text {env }}}{\partial n}\right) \mathrm{d} a+\int_{\mathcal{B}} \gamma \dot{\varphi} \mathrm{d} v
$$

for the generalized Swift-Hohenberg Eq. (53) subject to the uncoupled zero-dissipation boundary conditions (74) and (75) for the microtraction $\xi_{\mathcal{S}}$ and hypermicrotraction $\sigma_{\mathcal{S}}$.

\subsection{Generalized phase-field-crystal equation}

For the generalized phase-field-crystal equation, we assume that (74) and (75) and either the assigned constituent flow condition (76) or the assigned chemical potential condition (77), with $\mu_{\text {env }}=$ constant hold on all of $\partial \mathcal{B}$.

If (74), (75), and (76) hold on $\partial \mathcal{B}$, we see from (57) that the constituent-content balance for $\mathcal{B}$ becomes

$$
\overline{\int_{\mathcal{B}} \varphi \mathrm{d} v}=\int_{\partial \mathcal{B}} \jmath_{\text {env }} \mathrm{d} a+\int_{\mathcal{B}} s \mathrm{~d} v .
$$

Furthermore, proceeding as in the derivation of (93) but starting with (90) instead of (88) and using not only (74) and (75) but also (76), we obtain the identity

$$
\begin{aligned}
\overline{\int_{\mathcal{B}} \psi \mathrm{d} v}= & \int_{\partial \mathcal{B}}\left(\xi_{\mathrm{env}} \dot{\varphi}+\sigma_{\mathrm{env}} \frac{\partial \dot{\varphi}}{\partial n}+\mu \jmath_{\mathrm{env}}\right) \mathrm{d} a \\
& +\int_{\mathcal{B}}(\gamma \dot{\varphi}+\mu s) \mathrm{d} v \\
& -\int_{\mathcal{B}} \operatorname{grad} \mu \cdot \boldsymbol{M} \operatorname{grad} \mu \mathrm{d} v,
\end{aligned}
$$

where the dependence of the mobility tensor $\boldsymbol{M}$ on $\left(\varphi, \operatorname{grad} \varphi, \operatorname{grad}^{2} \varphi, \mu, \operatorname{grad} \mu\right)$ is again suppressed. Recalling that (73) ${ }_{1-3}$ apply on $\partial \mathcal{B}$ and the positivity condition (65) for $\boldsymbol{M}$, we thus deduce an energy-decay relation

$$
\begin{aligned}
\overline{\int_{\mathcal{B}} \psi \mathrm{d} v} \leq & \int_{\partial \mathcal{B}}\left(\xi_{\text {env }} \dot{\varphi}_{\text {env }}+\sigma_{\text {env }} \frac{\partial \dot{\varphi}_{\text {env }}}{\partial n}+\mu_{\text {env }} \jmath_{\text {env }}\right) \mathrm{d} a \\
& +\int_{\mathcal{B}}(\gamma \dot{\varphi}+\mu s) \mathrm{d} v
\end{aligned}
$$

for the generalized phase-field-crystal equations (66)(67) subject to the uncoupled zero-dissipation boundary conditions (74), (75), and (76) for the microtraction $\xi_{\text {env }}$, hypermicrotraction $\sigma_{\text {env }}$, and normal component $\jmath \cdot \boldsymbol{n}$ of the constituent flux $\jmath$.

If (74), (75), and (77) hold, the constituent-content balance (57) applies with $\mathcal{P}=\mathcal{B}$. Furthermore, proceeding as in the derivation of (96) while taking into consideration the assumption that

$$
\mu_{\mathrm{env}}=\mathrm{constant} \text {, }
$$

we obtain the identity 


$$
\begin{aligned}
& \overline{\int_{\mathcal{B}}\left(\psi-\mu_{\text {env }} \varphi\right) \mathrm{d} v}=\int_{\partial \mathcal{B}}\left(\xi_{\text {env }} \dot{\varphi}+\sigma_{\text {env }} \frac{\partial \dot{\varphi}}{\partial n}\right) \mathrm{d} a \\
& +\int_{\mathcal{B}}\left(\gamma \dot{\varphi}+\left(\mu-\mu_{\text {env }}\right) s\right) \mathrm{d} v \\
& -\int_{\mathcal{B}} \operatorname{grad} \mu \cdot \boldsymbol{M} \operatorname{grad} \mu \mathrm{d} v
\end{aligned}
$$

and, thus, again taking advantage of (65) and (73) $)_{1,2}$, arrive at the energy-decay relation

$$
\begin{gathered}
\int_{\mathcal{B}}\left(\psi-\mu_{\text {env }} \varphi\right) \mathrm{d} v \leq \int_{\partial \mathcal{B}}\left(\xi_{\text {env }} \dot{\varphi}_{\text {env }}+\sigma_{\text {env }} \frac{\partial \dot{\varphi}_{\text {env }}}{\partial n}\right) \mathrm{d} a \\
\quad+\int_{\mathcal{B}}\left(\gamma \dot{\varphi}+\left(\mu-\mu_{\text {env }}\right) s\right) \mathrm{d} v
\end{gathered}
$$

for the generalized phase-field-crystal equations (66)(67) subject to the uncoupled zero-dissipation boundary conditions (74) and (75) for the microtraction $\xi_{\text {env }}$ along with hypermicrotraction $\sigma_{\text {env }}$ and the assigned chemical potential condition (74) with $\mu_{\text {env }}=$ constant.

\section{Discussion of results}

In this work, we used the principle of virtual power and a free-energy imbalance to develop a secondgradient phase-field theory. In addition to the microtraction, microstress, and internal and external microforce densities that enter in the first-gradient phasefield theory by Fried and Gurtin [19], this theory involves a hypermicrostress and a corresponding hypermicrotraction. In our theory, the hypermicrostress and hypermicrotraction enter to account for power expenditures associated with temporal variations of the second spatial gradient of the phase field. The microforce balance (23) that arises from the principle of virtual power (10) in conjunction with the thermodynamically compatible constitutive relations (51) and (52) yields a family of evolution equations that encompasses and broadly generalizes the classical Swift-Hohenberg equation. When the phase field represents the volume fraction of a conserved constituent, a suitably augmented version (62) of the freeenergy imbalance yields constitutive relations (51) and (63) which, in conjunction with the microforce balance (23) determines the chemical potential as a functional of the phase field. Together with the constituent-content balance (58) and the constitutive relation (64) for the constituent flux yields a family of evolution equations that encompass and broadly generalizes the phase-field-crystal equation. The first-gradient phase-field theory of Fried and Gurtin [19] is recovered if higher-order terms, represented by the hypermicrotraction and hypermicrostress, arising from the second-gradient of the phase field are neglected. We also derived thermodynamically consistent boundary conditions (74)-(77) for our two families of evolution equations and associated energydecay relations. For our generalization of the SwiftHohenberg equation, the microtraction and hypermicrotraction are prescribed over the entire boundary of the region upon which the phase field is defined. For our generalization of the phase-field-crystal equation, the normal component of the constituent flux and the chemical potential are also prescribed on complementary portions of the boundary. In this case, we present two alternative energy decay relations, one corresponding to situations where only the normal component of the constituent flux is prescribed and the other corresponding to situations where the system is in contact with a reservoir of uniform chemical potential.

The framework presented here can be extended to incorporate other physical effects, such as deformation or thermal transport, and thus provides a platform for developing theories in which the phase field evolves in tandem with other independent kinematic or thermodynamic fields. Another possible enhancement, closely aligned with Gurtin's [20] derivation of the viscous Allen-Cahn equation, would involve accounting for viscous contributions to the microstress and hypermicrostress, yielding further generalizations of the Swift-Hohenberg and phase-field-crystal equations. For brevity, we leave such developments for future consideration.

Acknowledgements All authors thank the referees for their constructive suggestions. The corresponding author gratefully acknowledges the support from the Okinawa Institute of Science and Technology Graduate University with subsidy funding from the Cabinet Office, Government of Japan. This publication was also made possible in part by the CSIRO Professorial Chair in Computational Geoscience at Curtin University and the Deep Earth Imaging Enterprise Future Science Platforms of the Commonwealth Scientific Industrial Research Organisation, CSIRO, of Australia. This project received funding from the European Union's Horizon 2020 research and innovation 
programme under Marie Sklodowska-Curie Grant agreement No. 777778 (MATHROCKS). The Curtin Corrosion Centre and the Curtin Institute for Computation kindly provided ongoing support.

\section{Compliance with ethical standards}

Conflict of interest The authors declare that they have no conflict of interest.

Open Access This article is licensed under a Creative Commons Attribution 4.0 International License, which permits use, sharing, adaptation, distribution and reproduction in any medium or format, as long as you give appropriate credit to the original author(s) and the source, provide a link to the Creative Commons licence, and indicate if changes were made. The images or other third party material in this article are included in the article's Creative Commons licence, unless indicated otherwise in a credit line to the material. If material is not included in the article's Creative Commons licence and your intended use is not permitted by statutory regulation or exceeds the permitted use, you will need to obtain permission directly from the copyright holder. To view a copy of this licence, visit http://creativecommons.org/licenses/by/4.0/.

\section{References}

1. Swift J, Hohenberg PC (1977) Hydrodynamic fluctuations at the convective instability. Phys Rev A 15(1):319-328

2. Burke J, Knobloch E (2007) Homoclinic snaking: structure and stability. Chaos 17(3):037102-1-15

3. Golovin AA, Nepomnyashchy AA (2003) Disclinations in square and hexagonal patterns. Phys Rev E 67(5):056202$1-7$

4. Galla T, Moro E (2003) Defect formation in the SwiftHohenberg equation. Phys Rev E 67(3):035101

5. Boyer D, Viñals J (2001) Domain coarsening of stripe patterns close to onset. Phys Rev E 64(5):6050101(R)-1-4

6. Crawford C, Riecke H (1999) Oscillon-type structures and their interaction in a Swift-Hohenberg model. Physica D 129(1):83-92

7. Simeone D, Thorogood GJ, Murphy GL, Forestier A, Garcia P, Luneville L (2019) Radiation-induced micro-structures as ground states of a Swift-Hohenberg energy functional. J Appl Phys 125(6):065103-1-7

8. Ramakrishnan TV, Yussouff M (1979) First-principles order-parameter theory of freezing. Phys Rev B 19(5):2775-2794

9. Elder KR, Grant M (2004) Modeling elastic and plastic deformations in nonequilibrium processing using phase field crystals. Phys Rev E 70:051605

10. Stefanovic P, Haataja M, Provatas N (2009) Phase field crystal study of deformation and plasticity in nanocrystalline materials. Phys Rev E 80(4):046107-1-10

11. Löwen H (2010) A phase-field-crystal model for liquid crystals. J Phys: Condens Matter 22(36):364105-1-6

12. Gránásy L, Tegze G, Tóth GI, Pusztai T (2011) Phase-field crystal modelling of crystal nucleation, heteroepitaxy and patterning. Phil Mag 91(1):123-149
13. Yua Y-M, Backofen R, Voigt A (2011) Morphological instability of heteroepitaxial growth on vicinal substrates: A phase-field crystal study. J Cryst Growth 318(1):18-22

14. Berry J, Grant M (2014) Phase-field-crystal modeling of glass-forming liquids: spanning time scales during vitrification, aging, and deformation. Phys Rev E 89(6):062303$1-10$

15. Seymour M, Provatas N (2016) Structural phase field crystal approach for modeling graphene and other two-dimensional structures. Phys Rev B 93(3):035447-1-8

16. Yamanaka A, McReynolds K, Voorhees PW (2017) Phase field crystal simulation of grain boundary motion, grain rotation and dislocation reactions in a BCC bicrystal. Acta Mater 133:160-171

17. Skaugen A, Angheluta L, Viñals J (2018) Dislocation dynamics and crystal plasticity in the phase-field crystal model. Phys Rev B 97(5):054113-1

18. Provatas N, Dantzig JA, Athreya B, Chan P, Stefanovic P, Goldenfeld N, Elder KR (2007) Using the phase-field crystal method in the multi-scale modeling of microstructure evolution. JOM J Miner Metals Mater Soc 59(7):83-90

19. Fried E, Gurtin ME (1993) Continuum theory of thermally induced phase transitions based on an order parameter. Phys D 68(3-4):326-343

20. Gurtin ME (1996) Generalized Ginzburg-Landau and CahnHilliard equations based on a microforce balance. Phys D 92(3-4):178-192

21. Gurtin ME (2002) A gradient theory of single-crystal viscoplasticity that accounts for geometrically necessary dislocations. J Mech Phys Solids 50(1):5-32

22. Toupin RA (1962) Elastic Materials with Couple-stresses. Arch Ration Mech Anal 11(1):385-414

23. Toupin RA (1964) Theories of elasticity with couple-stress. Arch Ration Mech Anal 17(2):85-112

24. Germain P (1973) La méthode des puissances virtuelles en mécanique des milieux continus, I: Théorie du second gradient. J Mécanique 12(2):235-274

25. Antman SS, Osborn JE (1979) The principle of virtual work and integral laws of motion. Arch Ration Mech Anal 69(3):231-262

26. Del Piero G (2009) On the method of virtual power in continuum mechanics. J Mech Mater Struct 4(2):281-292

27. Fried E, Gurtin ME (2007) Thermomechanics of the interface between a body and its environment. Continuum Mech Thermodyn 19(5):253-271

28. Duda FP, Sarmiento A, Fried E (2019) Phase fields, constraints, and the Cahn-Hilliard equation. (Submitted)

29. Fried E, Gurtin ME (2006) Tractions, balances, and boundary conditions for nonsimple materials with application to liquid flow at small-length scales. Arch Ration Mech Anal 182(3):513-554

30. Coleman BD, Noll W (1963) The thermodynamics of elastic materials with heat conduction and viscosity. Arch Ration Mech Anal 13(1):167-178

Publisher's Note Springer Nature remains neutral with regard to jurisdictional claims in published maps and institutional affiliations. 